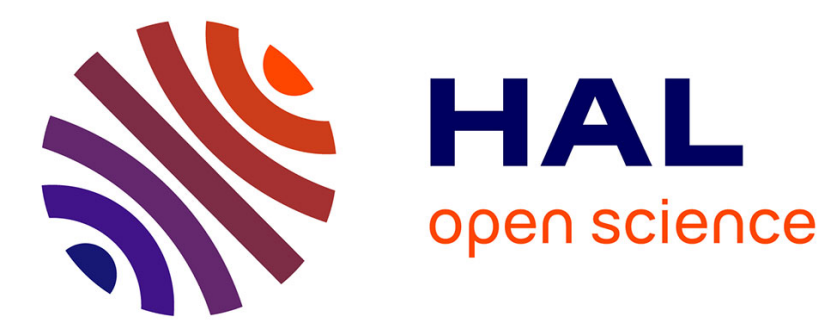

\title{
Dynamical holographic Moirés in a TEM
}

Christophe Gatel, Florent Houdellier, Etienne Snoeck

\section{To cite this version:}

Christophe Gatel, Florent Houdellier, Etienne Snoeck. Dynamical holographic Moirés in a TEM. Journal of Physics D: Applied Physics, 2016, 49 (32), pp.324001. 10.1088/0022-3727/49/32/324001 . hal-01707046

\section{HAL Id: hal-01707046 \\ https://hal.science/hal-01707046}

Submitted on 12 Feb 2018

HAL is a multi-disciplinary open access archive for the deposit and dissemination of scientific research documents, whether they are published or not. The documents may come from teaching and research institutions in France or abroad, or from public or private research centers.
L'archive ouverte pluridisciplinaire HAL, est destinée au dépôt et à la diffusion de documents scientifiques de niveau recherche, publiés ou non, émanant des établissements d'enseignement et de recherche français ou étrangers, des laboratoires publics ou privés. 


\title{
Dynamical Holographic Moirés in a TEM
}

\author{
C. Gatel ${ }^{\mathrm{a}, \mathrm{b}, *}$, F. Houdellier ${ }^{\mathrm{a}, \mathrm{b}}$, E. Snoeck $\mathrm{k}^{\mathrm{a}, \mathrm{b}}$ \\ ${ }^{a}$ CEMES-CNRS, 29 rue Jeanne Marvig, F-31055 Toulouse, France. \\ ${ }^{b}$ Université Paul Sabatier, Toulouse, France.
}

\begin{abstract}
A new electron interferometry method has been developed and implemented in a transmission electron microscope to quantitatively analyse magnetic and electric properties emanating from objects using holograms free of artifacts and with a frequential sensitivity. This method, called Dynamical Holographic Moirés (DHM ), is based on the double-exposure technique consisting in the superimposition of two different holograms. We improved this technique by acquiring the superimposed holograms for two well-defined excitation states of the sample and with a control of the superimposition frequency. The variations of magnetic and electrostatic fields between both excitation states can then be extracted directly from the amplitude part of the so-called interferogram. We demonstrate the efficiency of this method by studying quantitatively the magnetic field generated by a Hard Disk Drive writing head excited by a DC and an AC current. Double exposure measurements have also been performed to study in situ electrostatic properties of a biased carbon nanocone tip. Our method opens the route to dynamical studies using the unique combination of nanoscale resolution
\end{abstract}

*. Corresponding author

Email address: gatel@cemes.fr (C. Gatel)

Preprint submitted to J. Phys. D

13 juin 2016 
and electromagnetic sensitivity of electron interferometry.

Keywords: transmission electron microscopy, electron holography, phase reconstruction, electromagnetic fields, Holographic Moirés

\section{1. Introduction}

$2 \quad$ Off-axis Electron Holography (EH) [1] is a powerful interferometric method

3 carried out in Transmission Electron Microscopy (TEM) which enables to reco-

4 ver the phase shift that the fast electron beam experiences when interacting with

5 any electrostatic and/or magnetic field. This interaction between the incident

- electron beam and the electromagnetic field is described by the Aharonov-Bohm

7 effect $(\mathrm{AB})[2]$ using the following expression :

$$
\begin{aligned}
\phi(\mathbf{r}) & =C_{E} \int_{\infty} V(\mathbf{r}, z) d z-\frac{e}{\hbar} \int_{\infty} A_{z}(\mathbf{r}, z) d z \\
& =C_{E} \int_{\infty} V(\mathbf{r}, z) d z-\frac{\pi}{\Phi_{0}} \iint_{\infty} B_{\perp}(\mathbf{r}, z) d r d z
\end{aligned}
$$

8 where :

9 $\quad \mathbf{r}$ and $z$ are the $2 \mathrm{D}$ vector in the object/conjugated planes and the coordi-

10 nates along the optical axis respectively

$C_{E}$ is an electron wavelength related constant

$V$ is the electrostatic potential

$\phi_{0}$ is the magnetic flux quantum equal to $\frac{h}{2 e}$ where $e$ and $h$ are respectively the elementary charge and the Planck constant. $\phi_{0} \approx 2 \cdot 06810^{-15} W b$.

$A_{z}$ is the component of the magnetic potential vector parallel to the electron beam and is thus linked to magnetic induction gradients in the image 
17 plane.

${ }_{18} B_{\perp}$ is the component of the magnetic induction perpendicular to both $r$ and

19

Since the AB effect was experimentally been demonstrated using off-axis EH

21 by Tonomura [1], electron interferometry was used as an efficient tool for the 22 quantitative mapping of electrostatic and magnetic fields appearing within and

23 around a sample with a nanometer spatial resolution $[3,4,5,6,7,8,9,10]$.

24 EH has also been proved to be sensitive to the atomic displacement (and so the

25 strain fields) by studying the phase shift between diffracted beams through the

26 Dark-Field Electron Holography technique [11, 12] which is partially described

27 as a Moirés method. A Moirés pattern consists on interference fringes resulting

28 of the superimposition of two spatial periodic structures. As a result, a modu-

29 lation of the image intensity appears within the pattern. A simple distinction

30 between Moirés pattern and EH can be draw by the part of the wave under

31 interest : Moirés fringes appear as changes in the amplitude of the exit electron

32 wave while $\mathrm{EH}$ aims at retrieving the full exit wave, mostly focusing on its phase

33 part.

${ }_{34}$ Moirés method has intensively been used in TEM for decades for the study of

35 lattices deformation : stacking faults [13], crystal orientation [14], dislocations

36 [15], graphene stacks misalignments $[16,17,18]$, thickness estimation [19] are

37 some of the structural properties that can been studied using Moirés patterns.

38 More recently Moirés method has been implemented in high resolution STEM

39 [20] : the STEM Moirés fringes for a regularly periodic lattice arise when the 
40

41 crystalline or to a multiple of it.

42 The technique described in this paper combines Electron Holography and Moirés

43 patterns to improve the field sensitivity achievable in TEM through a frequency

${ }_{44}$ study and to develop the so-called Dynamical Holographic Moirés (DHM ) me-

45 thod. The basic idea of DHM is to superimpose two holograms obtained on a

46 same area of an object which is periodically stated in two different but control-

47 led configurations (either electrostatic or magnetic). The overlapping of both

48 holograms gives rise to Moirés contrasts in the final amplitude image carried

49 out by the fringes of the holograms.

so This idea already proposed in optical interferometry is based on the so-called

${ }_{51}$ double exposure technique (or holographic interferometry) and was formerly

52 used in a photonic bench. As examples of application, it enabled to access va-

53 riations of gas distribution during an arc discharge or to the macroscopic de-

54 formation of a given objects under external stress [21, 22]. It was then firstly

55 implemented in a electron microscope 30 years ago [23] and applied to off-axis

56 EH experiments on electromagnetic fields [24, 25, 26].

57 The double exposure technique presents different advantages. First at all

s8 the signal can be directly visualized and interpreted from the obtained image

59 by observing the modulation of the amplitude of fringes. Secondly it is not

6o necessary to correct the DHM pattern for any perturbations of the reference

${ }_{61}$ wave (as it has to be done for $\mathrm{EH}$ ) as these perturbations do not contribute

62 to the modulated amplitude if they remain the same for both holograms which 
${ }_{63}$ will be overlapped. Finally the Moirés contrasts linked to the relative signal

64 between both holograms are not influenced by the distortions originating from

65 the microscope setup (projector lens, camera,...) as they remain identical for all

66 images recorded at the same magnification. These advantages explain why the

67 use of such a technique was initially mandatory for simply and quantitatively

${ }_{68}$ mapping electromagnetic information at a large scale of pure phase objects.

69 An exhaustive and complete review of this technique can be found in ref [27].

70 Double exposure EH has been further developed by various groups to improve

71 the phase sensitivity by implementing various wavefront tilting [28, 29] or inter-

72 fering waves number $[30,31]$.

73 However, the main drawback of this method is a lower spatial resolution and a

74 lower sensitivity to weak signals compared to the ones obtained in off-axis EH.

75 In addition, the superimposition between both holograms requires to necessary

76 record the holograms with identical conditions and to control the signal change

77 between them. In addition, the emergence of computational hologram treatment

78 as well as the great improvement of CCD cameras with high pixels density, fast

79 acquisition rate and high pixel dynamic turned out this technique less used

so with respect to achievable modern methods (Fast Fourier treatment) which are

81 less time consuming for an improved sensitivity. Nevertheless, browsing the nu-

82 merous techniques commonly used in industrial interferometric holography, it

83 appears that the double exposure method is somehow the preliminary step to-

${ }_{84}$ wards dynamical characterization tools like the so-called real time and time

${ }_{85}$ average holographic interferometry [32]. 
${ }_{87}$ The purpose of this paper is to show the implementation of the time-average

s8 holographic interferometry in a TEM using the combination of off-axis EH and

89 in situ techniques. We highlight in the following the DHM capabilities with

9o two different in situ EH experiments : the first concerns the measurement of

91 magnetic induction generated by a writer pole of a Hard Disk Drive (HDD) as a

92 function of the $\mathrm{AC}$ current sent into its coils and the second is the electrostatic

93 field emanating from a biased carbon nanotube studied in situ by EH.

\section{2. Methods}

95 The DHM method is depicted by an illustration using the calculated elec-

96 trostatic field from a virtual charged sphere. Let us consider that the charge

97 state of such a sphere can be modulated between a positive and negative state.

98 The only feature required in our experiment is to be able to switch the charge

99 state of the sphere without any other modification of the optical system during the acquisition time. We define two coherent electron waves $\psi_{l, r}(\mathbf{r}, s)=$ $101 \quad A_{l, r}(\mathbf{r}, s) \exp \left(i \phi_{l, r}(\mathbf{r}, s)\right)$ passing on the left $(l)$ and the right $(r)$ side of a Möl-

lenstedt biprism which can be overlapped by applying a suitable voltage on the biprism. $s$ corresponds only to the object contribution to the phase (e.g. the charge of the virtual sphere). The resulting interference pattern (i.e. the hologram) contains the phase difference between the two beams $\Delta \phi(\mathbf{r}, s)=$ $\phi_{l, s}(\mathbf{r}, s)-\phi_{r, s}(\mathbf{r}, s)$ but also the phase distortions coming from the microscope setup $\phi_{\mu}(\mathbf{r})$. Fig. 1.b presents $\Delta \phi(\mathbf{r}, s)+\phi_{\mu}(\mathbf{r}, s)$ for a simulated positive charge 

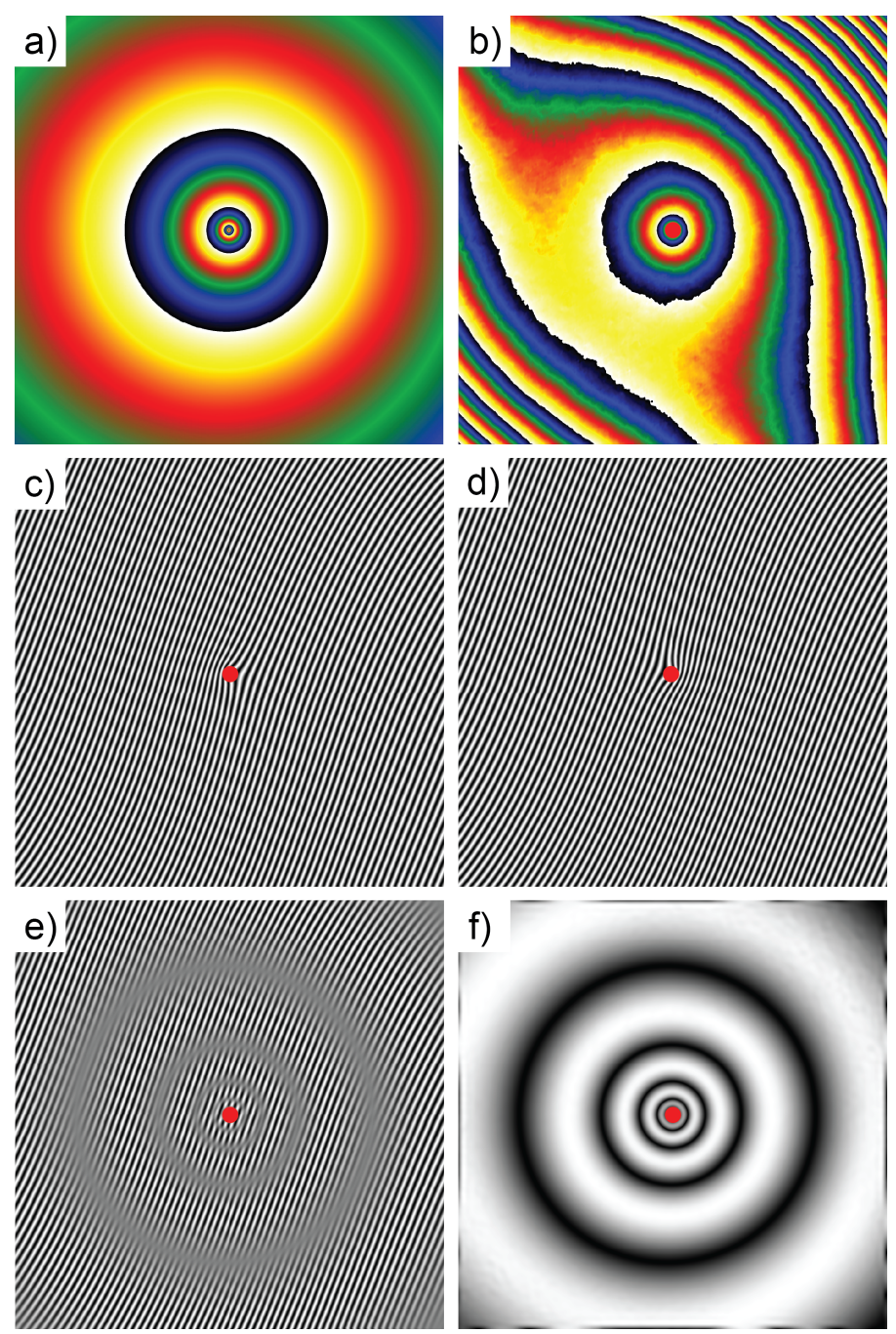

Figure 1: a) Phase image simulation of a sphere with a positive charge of $100 e$ (field of view equal to $1 \mu \mathrm{m})$. b) Phase image simulation of the same sphere including experimental phase distortions due to the microscope setup (projector lens and CCD camera). c) and d) Simulated holograms for a positive and negative charge respectively. e) Double exposure hologram (interferogram) corresponding to the addition of the two previous hologram. f) Electrostatic map obtained from the amplitude analysis of the interferogram in (f). 


$$
I_{H}(\mathbf{r}, s)=\left|A_{l}(\mathbf{r}, s)\right|^{2}+\left|A_{r}(\mathbf{r}, s)\right|^{2}+2 A_{l}(\mathbf{r}, s) A_{r}(\mathbf{r}, s) \cos \left(\mathbf{k}_{\mathbf{0}} \cdot \mathbf{r}+\Delta \phi(\mathbf{r}, s)+\phi_{\mu}(\mathbf{r})\right)
$$

This hologram displays a fringe deformation coming from the electric field generated by the sphere. The phase difference between the two beams $\Delta \phi(\mathbf{r}, s)$ is usually extracted by Fourier filtering while the phase distortions $\phi_{\mu}(\mathbf{r})$ are removed recording a reference hologram in the vacuum or knowing the different contributions to the phase distortions (projector lens, CCD camera,...) [33]. The Fig. 1.d shows the same hologram for an opposite charge.

The recorded image using the double exposure method corresponds to a simple addition of the half intensity of both previous holograms where $s_{1}$ and $s_{2}$ describe two different charge states (here opposite states with $s_{1}=-s_{2}$ ) :

$$
\begin{aligned}
I_{D H M}(\mathbf{r}) & =\frac{1}{2}\left(I_{H}\left(\mathbf{r}, s_{1}\right)+I_{H}\left(\mathbf{r}, s_{2}\right)\right) \\
& =\left|A_{l}\left(\mathbf{r}, s_{1}\right)\right|^{2}+\left|A_{r}\left(\mathbf{r}, s_{2}\right)\right|^{2}+A_{l}\left(\mathbf{r}, s_{1}\right) A_{r}\left(\mathbf{r}, s_{2}\right)\left(\cos \left(\mathbf{k}_{\mathbf{0}} \cdot \mathbf{r}+\Delta \phi\left(\mathbf{r}, s_{1}\right)+\phi_{\mu}(\mathbf{r})\right)\right) \\
& \left.+\cos \left(\mathbf{k}_{\mathbf{0}} \cdot \mathbf{r}+\Delta \phi\left(\mathbf{r}, s_{2}\right)\right)+\phi_{\mu}(\mathbf{r})\right)
\end{aligned}
$$

which can be rewritten into

$$
\begin{aligned}
& I_{D H M}(\mathbf{r})=\left|A_{l}\left(\mathbf{r}, s_{1}\right)\right|^{2}+\left|A_{r}\left(\mathbf{r}, s_{2}\right)\right|^{2}+2 A_{l}\left(\mathbf{r}, s_{1}\right) A_{r}\left(\mathbf{r}, s_{2}\right) \\
& \quad \cos \left(\frac{\Delta \phi\left(\mathbf{r}, s_{1}\right)-\Delta \phi\left(\mathbf{r}, s_{2}\right)}{2}\right) \cos \left(\mathbf{k}_{\mathbf{0}} \cdot \mathbf{r}+\frac{\Delta \phi\left(\mathbf{r}, s_{1}\right)+\Delta \phi\left(\mathbf{r}, s_{2}\right)}{2}+\phi_{\mu}(\mathbf{r})\right),
\end{aligned}
$$


113 This simple mathematical description shows that the amplitude of the holo114 graphic fringes is modulated by the cosine of the phase difference $\Delta \phi\left(\mathbf{r}, s_{1}\right)-$ ${ }_{115} \Delta \phi\left(\mathrm{r}, s_{2}\right)$ between both superimposed states. This associated cosine term // ${ }_{116}\left|\cos \left(\frac{\Delta \phi\left(\mathbf{r}, s_{1}\right)-\Delta \phi\left(\mathbf{r}, s_{2}\right)}{2}\right)\right|$ then corresponds to the envelop function of these ho117 lographic fringes and is the origin of the Moirés contrasts. Note that this term 118 does not depend on the holographic fringe periodicity i.e. the applied voltage on the biprism. As depicted Fig. 1.a and Fig. 1.e, we can clearly observe the

133 holographic fringes between both superimposed holograms, i.e. a phase shift of ${ }_{134} 2 \pi$ coming from the presence of an electromagnetic field. For example, in case 
135

of magnetic induction only (no changes in the electrostatic contribution), it delimits a magnetic flux equal to $2 \Phi_{0}$. For an electric field, this distance represents a variation of the projected electrostatic potential (i.e. integrated along the electron path) of $\frac{2 \pi}{C_{E}}$. The experimental challenge to perform successfully such DHM experiment relies on the ability to quickly vary and control the electromagnetic field for making an addition of at least two defined states during the acquisition time, keeping the whole optical alignment stable without any change. Microelectronic devices whose state can be tuned in two configurations by an external alternative input of various frequencies are perfect systems to be studied by DHM . Any defined state can then be studied with any frequency.

\section{Results and discussion}

The major assumption made in the DHM description is the possibility to change and to control the magnetic or the electric state only of the sample under study. In a previous EH experiment we have successfully studied the magnetic field emanating from a Hard Disk Drive (HDD) writer pole [34]. In this previous paper, we developed a novel strategy that allows in situ imaging an operational write pole of a HDD slider in a TEM in normal working conditions.

The complete local hysteresis loop on each point of a large area has been obtained while the magnetic signals originating only from the write pole and those of the shield material have been separated and quantitatively analyzed changing the applied current through the write coils. The writing/erasing magnetic field 


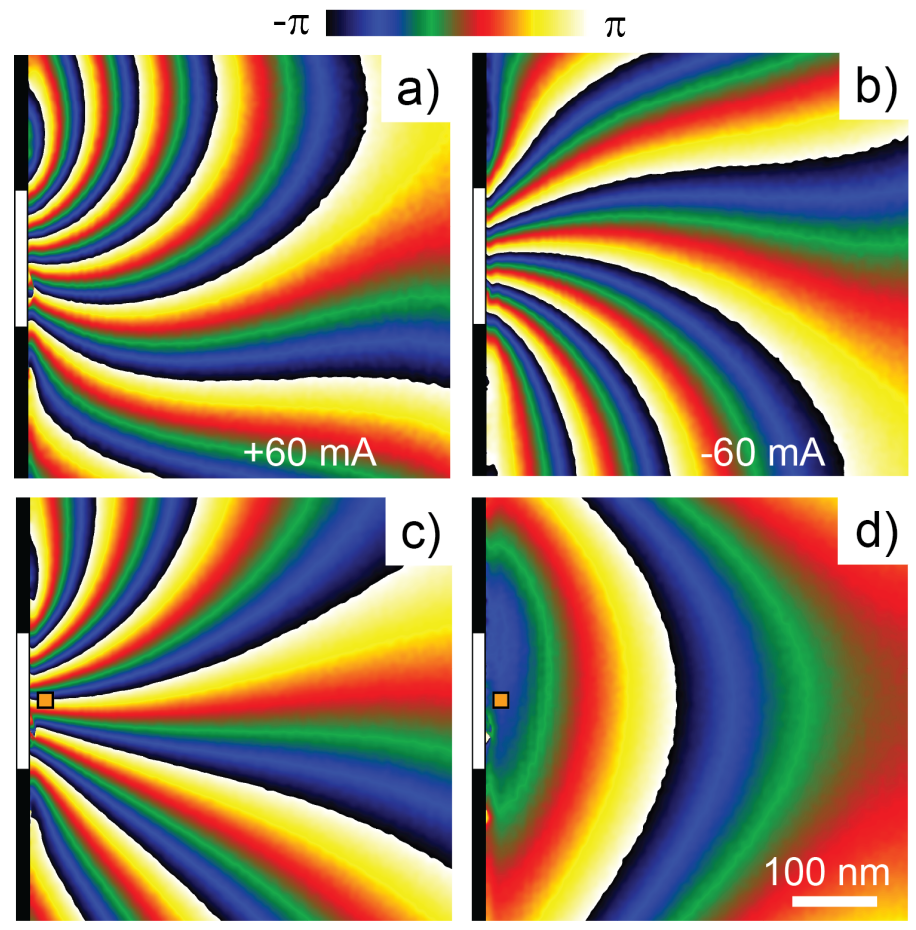

Figure 2: Off-axis electron holography performed on a HDD writing head reported in [34]. a and b) Wrapped magnetic phase images obtained for a current injected of $+60 \mathrm{~mA}$ and $-60 \mathrm{~mA}$ respectively. $\mathrm{c}$ and d) Half sum and half difference of the phase images displayed in $\mathrm{a}$ and $\mathrm{b}$ revealing respectively the pole and shield contributions 
${ }_{157}$ is generated by the current flowing in the coils intended to behave linearly with

158 the applied current without hysteresis when reversing the current to allow for 159 a fast and accurate writing and erasing of data. To precisely tailor the profile 160 of emanating magnetic field, the current carrying coils and bulk magnetic ma161 terial of the writer are shielded from the disk media. This also insures that the 162 magnetic environment experienced by the media is dominated by the write pole $\mathbf{1 6 3}$ and not from other surfaces of this three dimensional electromagnetic device.

164 These shields are composed of soft magnetic materials to amplify the magnetic 165 field gradients coming out of the write pole (more details on the HDD writer 166 part description in ref [34]). The slider was carefully prepared to be electrically 167 bounded on a dedicated TEM sample holder. We achieved to make a thin TEM 168 sample reducing the overall size of a HDD slider from $1.2 \mathrm{~mm}$ down to $30 \mu \mathrm{m}$ 169 by mechanically polishing while keeping intact the writer coils and the electrical contact pads. The in situ EH experiment was performed in Lorentz mode on 171 a Cs-corrected Tecnai F20 microscope operating at 200kV. The Möllenstedt bi172 prism, positioned parallel to the surface of the slider at a distance of about 600 $173 \mathrm{~nm}$, is used to create the interference pattern (applied voltage of $140 \mathrm{~V}$ giving 174 a fringes periodicity equal to $2 \mathrm{~nm}$ ). The resulting electron hologram then allows 175 extraction of the phase shift between the electron beam interacting with the 176 generated magnetic induction field in front of the write pole and a reference ${ }_{177}$ beam that passes far from it through an almost field-free region [34]. In this 178 experiment, the electron phase shift is only related to the magnetic induction 179 through the Aharanov-Bohm relation presented in the first part of this article 
as no electrostatic signal is present.

$$
\text { It is then possible to inject either a positive (Fig. 2.a) or a negative (Fig. 2.b) }
$$
DC current through the coil of the pole during EH experiment for phase analysis. The white rectangle on the middle left side is the write pole location. Stating that the magnetic flux generated is symmetric with respect to the current sign, simple half sum (Fig. 2.c) and half subtraction (Fig. 2.d) of two phase images obtained for opposite currents were performed to access to the intrinsic writer pole magnetic flux and the intrinsic magnetic shield contribution respectively. Using a complete set of excitation currents between $-60 m A$ and $+60 m A$, we are able to obtain a hysteresis loop along any direction of the magnetic flux being emitted by the pole and the shield for any location in the studied area. More details regarding this experiment as the influence of the weak signal in the reference wave can be found in [34]. Fig. 3.a presents the hysteresis loops of the horizontal component (i.e. perpendicular direction to the surface of the pole) of the projected magnetic inductions for the pole and the shield. They have been calculated from an area located in front of the write pole at a distance of $5 \mathrm{~nm}$ from its surface as marked by the orange square on the Fig. 2.c and Fig. 2.d. We can clearly observe that the shield contribution presents a rather constant and low value while the pole contribution shows an important variation of the projected magnetic induction. The highest value corresponding to the magnetic saturation of the projected induction at 118T.nm is reached for an applied current around $20 \mathrm{~mA}$. Finally Fig. 3.a demonstrates the absence of remnant and coercive fields that are both expected for a HDD read/write device. 

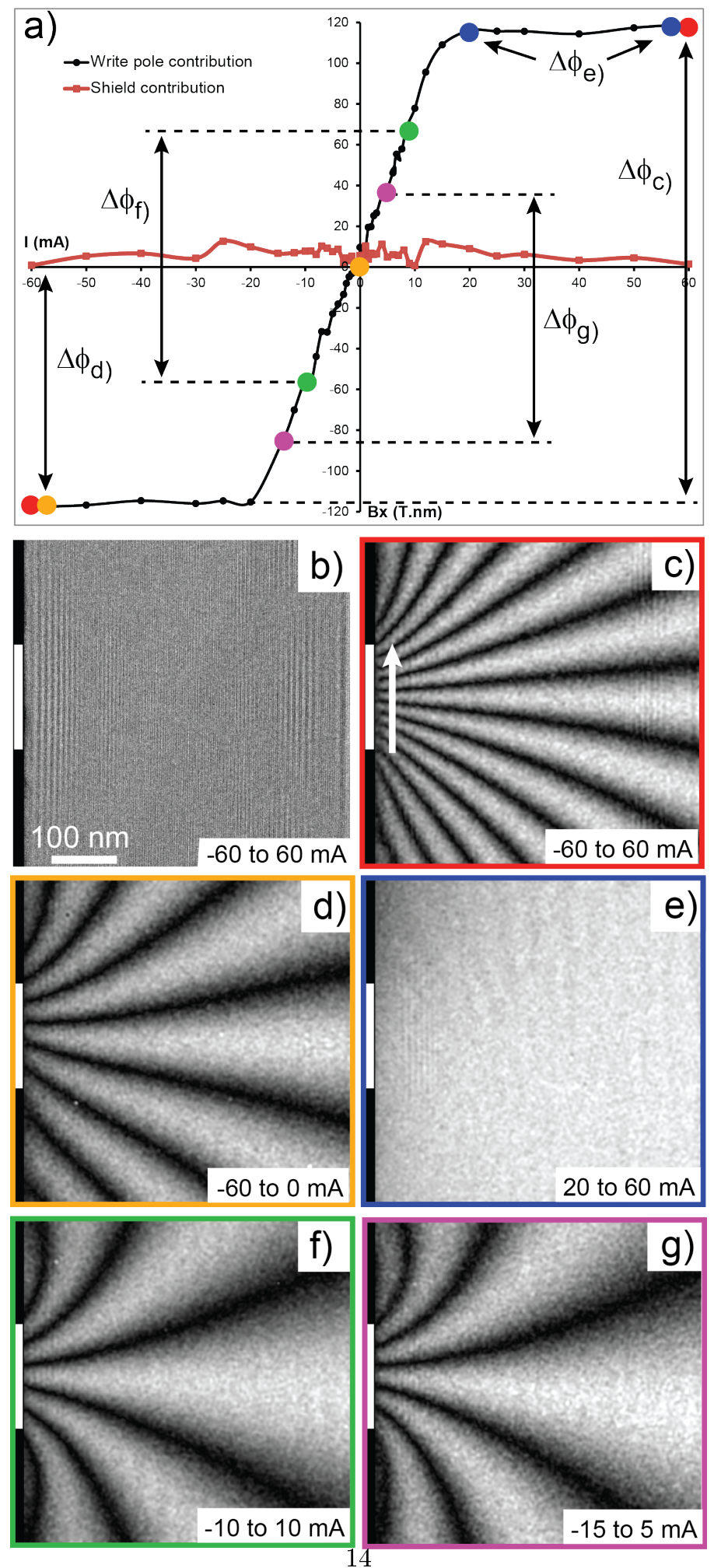

Figure 3: Time-Averaged holographic interferometry on the HDD writing head. a) Hysteresis loop of the horizontal magnetic induction component (perpendicular to the pole surface) for the writing pole (black) and the shield (red). Colored dots and corresponding $\Delta \phi_{i}$ correspond to the amplitude signal between the excitation states used in the following interferograms. b) 
${ }_{224}$ a total magnetic flux of $20 \phi_{0}$ i.e. $41.3610^{-15} \mathrm{~Wb}$ ${ }_{225}$ If the $\mathrm{AC}$ signal is set between -60 and $0 m A\left(\Delta \phi_{d}\right)$, the amplitude of the maemanating magnetic field oscillates between the two states defined by the AC square signal. The oscillation of the signal may then be tuned with different current values and frequencies during the acquisition time allowing to obtain interferograms by the DHM method. The amplitude part of these interferograms displayed in Fig. 3 corresponds to the ones obtained for the AC signal depicted with a color code on the hysteresis loop. The frequency was set at 1 $\mathrm{kHz}$ and the acquisition time at $4 \mathrm{~s}$ meaning that 4000 holograms were overlapped (2000 for each current value/magnetic state). We start our description with the case of an $\mathrm{AC}$ square signal between -60 and $+60 \mathrm{~mA} @ 1 \mathrm{kHz}$ which are the maximal values of injected current. The corresponding magnetic variation is represented on Fig. 3.a by $\Delta \phi_{c}$. The resulting interferogram directly recorded by the CCD camera is displayed Fig. 3.b. From the extracted amplitude image by Fourier analysis with a circular numerical mask giving a spatial resolution of $6 \mathrm{~nm}$ (Fig. 3.c), we clearly see that the Moirés pattern fits qualitatively with the intrinsic signal of the pole only as depicted in Fig. 2.c. This illustrates that the shield contribution, which remains constant (as well as any contant phase distortion coming from the microscope setup), is not contributing to the DHM amplitude and that DHM only reveals the magnetic changes associated to the $\mathrm{AC}$ square current. Approximately 10 white lines surrounded by dark lines emanating from the writer part can be deducted. They then correspond roughly to

When an AC square current is sent into the coils (instead a DC one), the 
241 holograms. This phase shift is equivalent to a magnetic flux variation of $2 \Phi_{0}$

$242 \quad\left(4.13610^{-15} \mathrm{~Wb}\right)$ through a surface perpendicular to the induction defined by a

243 rectangle with a width equal to the distance between minima and an infinite

244 length parallel to the electron beam. Dividing this flux by the distance between

245 minima amounts to obtain the integrated magnetic induction along the electron

246 path (unit T.nm) which is the physical parameter reported in the hysteresis

247 loop presented Fig. 3.a. From the red curve in Fig. 4.a, the distance between

248 two minima (centered on the writer pole) is measured to be about $18 \mathrm{~nm}$ gi- 
249 $261-15$ and $5 m A\left(\Delta \phi_{g}\right)$, we define the same magnetic variation than $\Delta \phi_{d)}$ since

ving a projected induction variation of 230 T.nm. This variation corresponds to twice the saturated magnetic induction $\left(\Delta \phi_{d)}\right.$ for -60 to $\left.+60 \mathrm{~mA}\right)$ : we have to divide by 2 to obtain the final value of the saturated induction equal to 115 T.nm. This value is very close to the one measured by classical off-axis EH and reported on the hysteresis loop. Using the blue curve with $\Delta \phi_{d)}$ as variation, the distance between two minima is now $36 \mathrm{~nm}$ which is twice the previous distance. The corresponding projected induction is then half of the previous one and so in agreement with the value obtained by off-axis EH. The yellow curve $\left(\Delta \phi_{e}\right)$ confirms the absence of real magnetic variation between the two states even in the presence of an important magnetic field.

If we now probe the signal within the hysteresis loop, keeping a constant $\mathrm{AC}$ current amplitude of $20 m A$, i.e. between -10 and $10 m A\left(\Delta \phi_{f}\right)$ and between the magnetic saturation is almost achieved for an applied current of $20 \mathrm{~mA}$. On the corresponding amplitudes images Fig. 3.f and Fig. 3.g, the same quantification of the emitted magnetic flux is obtained : 5 whites lines for a total magnetic flux of $10 \Phi_{0}$. The Fig. 4.b shows the nice superimposition of the corresponding quantitative profiles and illustrates the same variation of the projected magnetic induction at various levels equal to 115 T.nm. Some slight differences can however be noticed between the two amplitude images in Fig. 3.f and Fig. 3.g and the one between -60 and $0 m A$ in Fig. 3.d. These discrepancies are also visible at the extremities of the profiles. They can be explained studying which parts of the hysteresis loop contribute to the DHM pattern : the first two cases 
${ }^{272}\left(\Delta \phi_{f}\right)$ and $\left.\Delta \phi_{g}\right)$ are located on the linear variation of the magnetic flux with 273 the injected current while the last one includes the saturated part. Even if the 274 saturation state is reached, small variations in the magnetic flux can arise with 275 the overflow current and certainly the interaction between the fields produced 276 by the pole and the magnetic shield is different. Both effects may induce the 277 asymmetry while keeping valid the quantification of the magnetic flux that is 278 measured at the vicinity of the pole.

279 As a brief summary of these results, we claim that DHM allows to probe 280 all part of the hysteresis loop independently, without any complex data treat281 ment and in a qualitative way. In addition, the magnetic response of this device 282 under the application of an alternative current can be studied for different AC 283 frequencies. DHM allows then to study the frequency dependence of the system ${ }_{284}$ under consideration, as described below.

285

The behaviour of the HDD writer head is now studied as a function of fre287 quency with a fixed amplitude signal $(-60$ and $60 \mathrm{~mA}$ ). The frequency is tuned ${ }_{288}$ from $0.5 \mathrm{~Hz}$ to $50 \mathrm{kHz}$ keeping the acquisition time fixed to $4 \mathrm{~s}$. Two variations ${ }_{289}$ can thus be expected in that case : first at all the signal quality could be mod290 fied with the frequency as more holograms are added in a single interferogram 291 and secondly inductance effect could arise when increasing the frequency thus 292 modulating the magnetic flux. Four different interferograms for four different ${ }_{293}$ frequencies are depicted in Fig. 5. : no noticeable modification of the magnetic 294 flux spreading from the pole is observed : the shape shown on the amplitude 

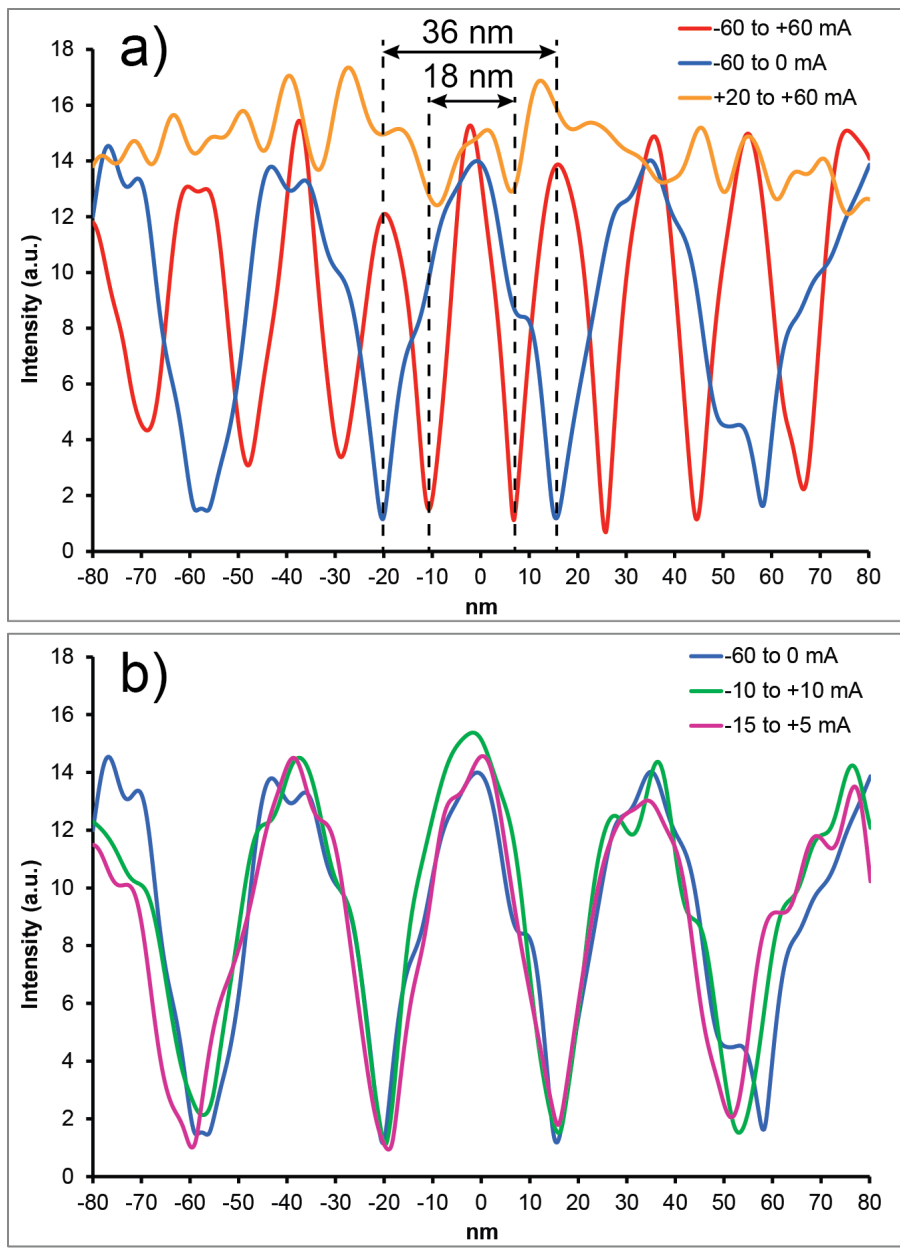

Figure 4: Profiles of interferograms extracted from Fig. 3.a at a distance of $5 \mathrm{~nm}$ from the surface (white arrow of Fig. 3.c). a) Profiles extracted from Fig. 3.c, d and e). b) Profiles extracted from Fig. 3.d, f and g). 

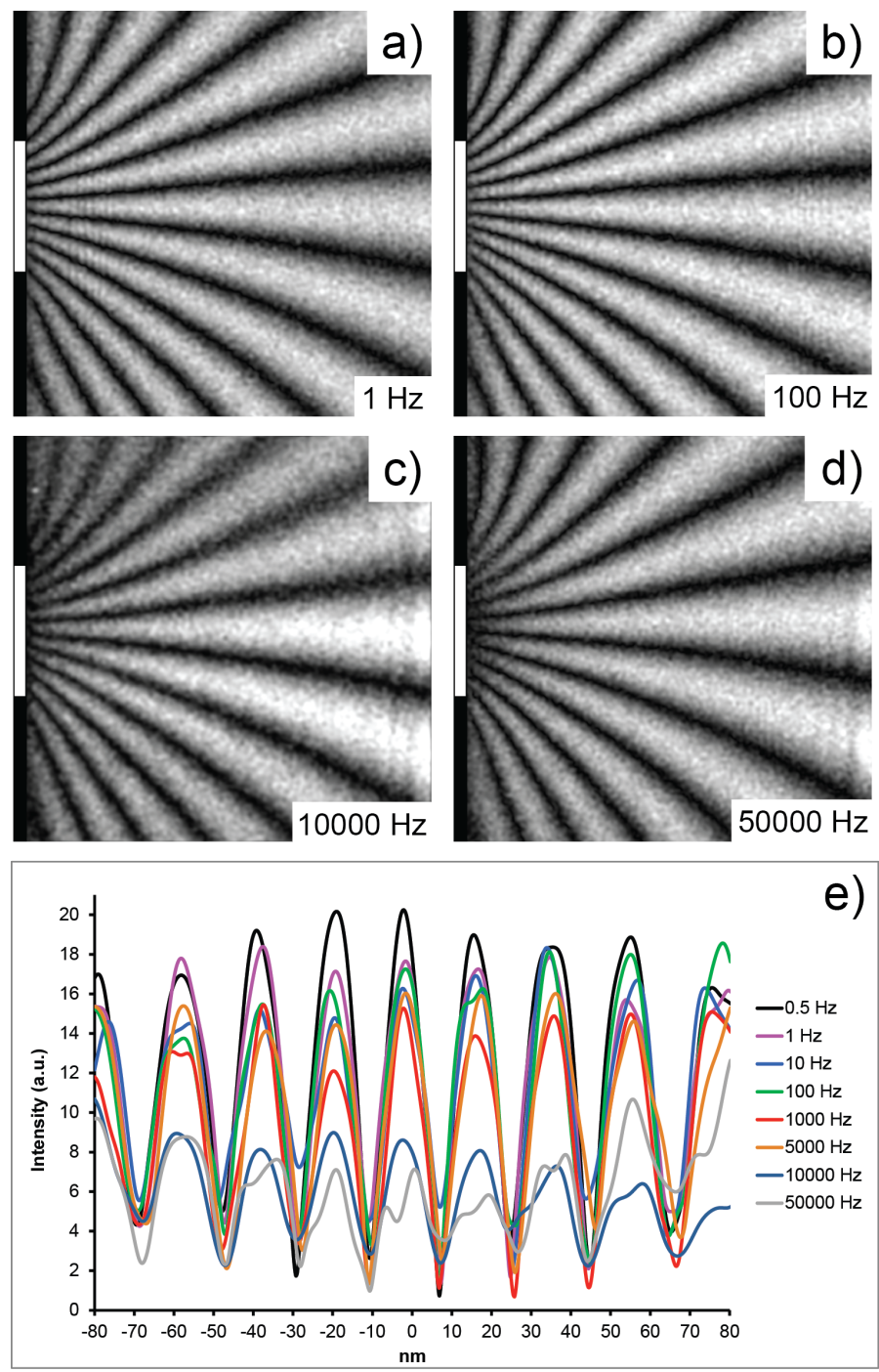

Figure 5: Frequency analysis on the interferograms at a fixed amplitude of $+\backslash-60 m A$. a - d) Frequency of $1 \mathrm{~Hz}, 100 \mathrm{~Hz}, 10 \mathrm{kHz}$ and $50 \mathrm{kHz}$ respectively. e) Profiles of interferogram (same as in Fig. 4. extracted from various frequency measurements). 

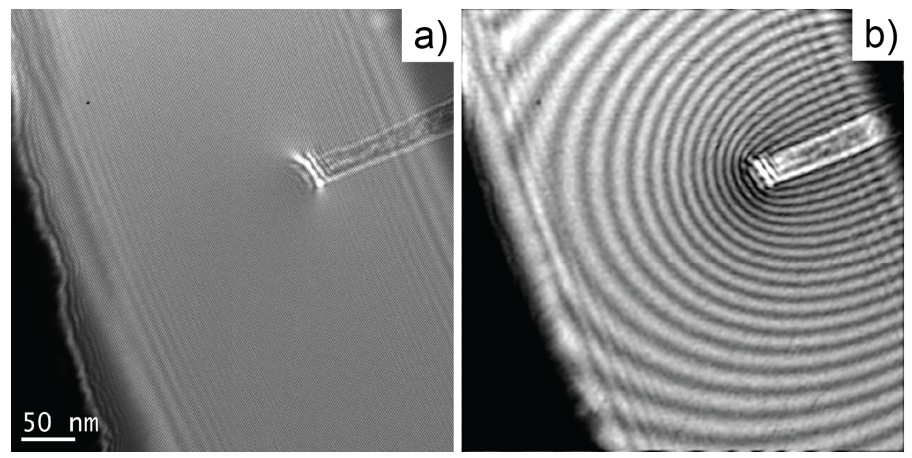

Figure 6: Double exposure holography on a field emitting Carbon Cone nanoTip. a) Raw hologram obtained for the emitting CCnT during its collapse. b) Amplitude extracted from a).

images (Fig. 5.a to Fig. 5.d) as well as the value of the projected induction deduced from profiles in Fig. 5.e remain the sames. We observed only an important decrease of the fringe amplitude close to the write pole for 'high-frequency' excitations $(\geqslant 10 \mathrm{kHz})$. This is supposed to be related to our experimental set up (sample holder, source meter and cables) that is not designed for such high frequency experiments producing possible decay of the electrical power and mechanical vibrationss. As it worth noting that a standard HDD writing head is supposed to work at a frequency of $2.4 \mathrm{GHz}$, a dedicated sample holder for high frequency signals should be used to explore such high frequency domain in order to observe and study local dumping due to the inductance effect.

We performed a last experiment on the HDD writer head changing the applied voltage on the biprism (i.e. the distance between the holographic fringes) keeping the others parameters constant. As expected no effect of the biprism voltage is observed : the periodicity of the holographic fringes does not interfere 
326

with the formation of the Moiré pattern.

The Fig. 6.illustrates the capability of DHM in the observation of other types of signal using other kind of stimuli. In a previous work we studied in situ the field emission process of Carbon Cone nanoTips (CCnT) [35]. During a particular Electron Holography experiment it turned out that the CCnT broke due to an arc discharge during the acquisition time of an hologram. The analyze of the amplitude of the resulting hologram (Fig. 6.a) evidences a nice pattern corresponding to the electrostatic potential changes when the tip is switched down from $+100 \mathrm{~V}$ to $0 \mathrm{~V}$ (Fig. $6 . \mathrm{b}$ ). On the contrary to the HHD writing head analysis previously described and for which time-average holographic interferometry was performed, that unintentional DHM experiment carried out on the CCnT is a simple double exposure experiment. Nevertheless it clearly evidences the performances of DHM experiments to study devices in which the electrostatic field can be switched between two states. It is however obvious that high AC electric field application in a TEM is a more complex task as it deviates easily the electron beam and is tedious to master particularly within the objective pole pieces of a TEM.

This last remark is in fact a general drawback of the DHM method : it requires to master the combined complexity of both the EH and in situ experimental constraints. Moreover DHM is only limited to the study of perfectly reversible processes for which the two states that are superimposed in the interferogram are fully reproducible. Finally the quantification of the interferogram 
332

${ }_{333}$ by off-axis EH. In addition, the appearance of the Moiré pattern is linked to a

334 sufficient variation of the signal allowing multiple of $2 \pi$ phase shift. This method

${ }_{335}$ is therefore not adapted to the study of weak signal variations. However DHM

336

337

338

339

340

${ }^{341}$ camera distortions). Therefore, the requested phase information is always mixed

342 with unexpected phase modulation arising from the microscope setup. These ar-

34 tifacts can be removed using a couple of holograms [36]. A second limitation of

34 off-axis EH may occur when no reference area of the studied field of view on the

345 phase image can be chosen to correct the slope arising from the Fourier ana-

346 lysis (misalignment between the center of the numerical mask and the carrier

${ }_{347}$ frequency). This problem can be encountered when studying a magnetic wall

348 and its leak field. The third limitation is that, like most of the TEM methods,

349 typical acquisition time for EH experiment is between hundred of milliseconds

350 to ten seconds, limiting the technique to pseudo-static studies. Dynamical stu-

${ }_{351}$ dies in TEM require the use of complex instruments $([37,38])$ where electron

352 pulses are generated by photo-emission processes thanks to the use of control-

353 led pulsed laser. Up to now the limited brigthness and poor spatial coherence of

${ }_{354}$ the ultrafast photocathodes used in these advanced Dynamical-TEMs (DTEMs) 

375 Similar analysis could be implemented to study electric fields. of instrumental developments toward high frequency signal injection).

\section{Conclusion}

does not permit to perform electron interferometry experiments on them. We claim that our new DHM method is addressing (at least part of) all these three points. We showed that phase distortions either coming from the microscope setup are not influencing DHM interferograms. Moreover DHM interferograms can be directly quantified without any needs of other image treatments. They can be obtained between different defined excitation states and used to study the resulting signal variation in terms of amplitude and distribution. Most of all, the possibility to tune the frequency opens the way to emphasis dynamical effects and damping functions such as inductance or capacitance (at the expand

We showed that this DHM technique raised the possibility of studying easily and quantitatively a system that is stated in two reproducible magnetic or electrostatic configurations and to extract quantitative information on these configurations without time consuming and complex electron hologram analysis.

This technique was demonstrated to overcome traditional EH limitations such as artifacts from the microscope setup or others static constant phase shift 73 contributions. We succeed in analyzing dynamically the behavior of a HDD 374 writing head and studying different part of the corresponding hysteresis loop. 
377 The authors acknowledge A. Masseboeuf for carefully reading and correcting

\section{Acknowledgments} the paper. The authors also acknowledge the European Union under the Seventh Framework Programme under a contract for an Integrated Infrastructure Initiative Reference 312483-ESTEEM2, the ANR EMMA 12-BS10-0013 project, the support of the French National Research Agency under the Investissement d Avenir program reference No. ANR-10-EQPX-38-01, and the Conseil Regional Midi-Pyrénées, the European FEDER within the CPER program and the

Labex NEXT through the MIME project for financial support.

\section{References}

[1] A. Tonomura, N. Osakabe, T. Matsuda, T. Kawasaki, J. Endo, S. Yano, H. Yamada, Evidence for aharonov-bohm effect with magnetic field completely shielded from electron wave, Physical Review Letters 56 (8) (1986) 792-795. doi :10.1103/PhysRevLett.56.792.

URL http://link.aps.org/doi/10.1103/PhysRevLett.56.792

[2] Y. Aharonov, D. Bohm, Significance of electromagnetic potentials in the quantum theory, Physical Review 115 (3) (1959) 485-491.

[3] A. Tonomura, Applications of electron holography, Reviews of Modern Physics 59 (3) (1987) 639-669. doi :10.1103/RevModPhys.59.639. URL http://link.aps.org/doi/10.1103/RevModPhys.59.639 
[4] P. A. Midgley, R. E. Dunin-Borkowski, Electron tomography and holography in materials science, Nat Mater 8 (4) (2009) 271-280. doi :10.1038/nmat2406.

URL http://dx.doi.org/10.1038/nmat2406

[5] M. Beleggia, T. Kasama, R. E. Dunin-Borkowski, M. Beleggia, T. Kasama, R. E. Dunin-Borkowski, The quantitative measurement of magnetic moments from phase images of nanoparticles and nanostructures - I. Fundamentals, Ultramicroscopy 110 (5) (2010) $\quad 425-432$. doi :10.1016/j.ultramic.2009.10.007.

[6] A. C. Twitchett, R. E. Dunin-Borkowski, P. A. Midgley, Quantitative Electron Holography of Biased Semiconductor Devices, Physical Review Letters 88 (23) (2002) 238302. doi :10.1103/PhysRevLett.88.238302.

URL http://link.aps.org/doi/10.1103/PhysRevLett.88.238302

[7] C. Gatel, A. Lubk, G. Pozzi, E. Snoeck, M. Hÿtch, Counting Elementary Charges on Nanoparticles by Electron Holography, Physical Review Letters 111 (2). doi :10.1103/PhysRevLett.111.025501.

URL http://link.aps.org/doi/10.1103/PhysRevLett.111.025501

[8] N. Biziere, C. Gatel, R. Lassalle-Balier, M. C. Clochard, J. E. Wegrowe, E. Snoeck, Imaging the Fine Structure of a Magnetic Domain Wall in a Ni Nanocylinder, Nano Letters 13 (5) (2013) 2053-2057. doi $: 10.1021 / \mathrm{nl} 400317 \mathrm{j}$.

URL http://dx.doi.org/10.1021/nl400317j 
[9] C. Gatel, F. J. Bonilla, A. Meffre, E. Snoeck, B. Warot-Fonrose, B. Chaudret, L.-M. Lacroix, T. Blon, Size-Specific Spin Configurations in Single Iron Nanomagnet : From Flower to Exotic Vortices, Nano Letters 15 (10) (2015) 6952-6957. doi :10.1021/acs.nanolett.5b02892.

URL http://pubs.acs.org/doi/10.1021/acs.nanolett.5b02892

[10] D. Reyes, N. Biziere, B. Warot-Fonrose, T. Wade, C. Gatel, Magnetic Configurations in $\mathrm{Co} / \mathrm{Cu}$ Multilayered Nanowires : Evidence of Structural and Magnetic Interplay, Nano Letters 16 (2) (2016) 1230-1236. doi :10.1021/acs.nanolett.5b04553.

URL http://dx.doi.org/10.1021/acs.nanolett.5b04553

[11] M. Hytch, F. Houdellier, F. Hue, E. Snoeck, Nanoscale holographic interferometry for strain measurements in electronic devices, Nature 453 (7198) (2008) 1086-1089. doi :10.1038/nature07049.

URL http://dx.doi.org/10.1038/nature07049

[12] M. Hytch, N. Cherkashin, S. Reboh, F. Houdellier, A. Claverie, Strain mapping in layers and devices by electron holography, Physica Status Solidi a-Applications and Materials Science 208 (3) (2011) 580-583, wOS :000288177600018. doi :10.1002/pssa.201000281.

[13] J. F. Goodman, Evidence from moiré patterns of packing faults in boron nitride crystals, Nature 180 (4583) (1957) 425-427. doi :10.1038/180425a0. URL http://www .nature. com/nature/journal/v180/n4583/abs/180425a0.html

[14] S. A. Nepijko, M. Klimenkov, M. Adelt, H. Kuhlenbeck, R. Schlögl, 
H.-J. Freund, Structural investigation of palladium clusters on $\gamma-$ alo3(111)/nial(110) with transmission electron microscopy, Langmuir 15 (16) (1999) 5309-5313. doi :10.1021/la981012p.

URL http://dx.doi.org/10.1021/la981012p

[15] G. A. Bassett, J. W. Menter, D. W. Pashley, Moiré patterns on electron micrographs, and their application to the study of dislocations in metals, Proceedings of the Royal Society of London. Series A, Mathematical and Physical Sciences 246 (1246) (1958) 345-368, articleType : research-article / Full publication date : Aug. 19, 1958 / Copyright (C) 1958 The Royal Society.

URL http://www.jstor.org/stable/100499

[16] F. E. Fujita, K. Izui, Observation of lattice defects in graphite by electron microscopy, part i, Journal of the Physical Society of Japan 16 (2) (1961) 214-227. doi :10.1143/JPSJ.16.214.

URL http://jpsj.ipap.jp/link?JPSJ/16/214/

[17] H. Shang, H. Xie, H. Zhu, F. Dai, D. Wu, W. Wang, Y. Fang, Investigation of strain in individual multi-walled carbon nanotube by a novel moiré method, Journal of Materials Processing Technology 170 (1-2) (2005) 108-111. doi :10.1016/j.jmatprotec.2005.04.089.

URL http://www.sciencedirect.com/science/article/pii/S0924013605005261

[18] J. H. Warner, M. H. Rümmeli, T. Gemming, B. Büchner, G. A. D. Briggs, Direct imaging of rotational stacking faults in few layer graphene, Nano 
Letters 9 (1) (2009) 102-106. doi :10.1021/nl8025949.

URL http://dx.doi.org/10.1021/n18025949

[19] M. Valamanesh, C. Langlois, D. Alloyeau, E. Lacaze, C. Ricolleau, Combining moiré patterns and high resolution transmission electron microscopy for in-plane thin films thickness determination, Ultramicroscopy 111 (2) (2011) 149-154. doi :10.1016/j.ultramic.2010.10.017.

URL http://www.sciencedirect.com/science/article/pii/S0304399110002792

[20] S. Kim, S. Lee, Y. Oshima, Y. Kondo, E. Okunishi, N. Endo, J. Jung, G. Byun, S. Lee, K. Lee, Scanning moiré fringe imaging for quantitative strain mapping in semiconductor devices, Applied Physics Letters 102 (16) (2013) 161604. doi :10.1063/1.4803087.

URL http://scitation.aip.org/content/aip/journal/apl/102/16/10.1063/1.4803087

[21] L. O. Heflinger, R. F. Wuerker, R. E. Brooks, Holographic interferometry, Journal of Applied Physics 37 (2) (1966) 642-649. doi :10.1063/1.1708231. URL http://scitation.aip.org/content/aip/journal/jap/37/2/10.1063/1.1708231

[22] R. L. POWELL, K. A. STETSON, Interferometric vibration analysis by wavefront reconstruction, Journal of the Optical Society of America 55 (12) (1965) 1593-1597. doi :10.1364/JOSA.55.001593.

URL http: //www .opticsinfobase .org/abstract.cfm?URI=josa-55-12-1593

[23] S. Fu, J. Chen, Z. Wang, H. Cao, Experimental investigation of electron interference and electron holography, Optik - International Journal for 
Light and Electron Optics 76 (2) (1987) 45-47.

URL http://www.sciencedirect.com/science/article/pii/S0030402612008881

[24] S. Frabboni, G. Matteucci, G. Pozzi, Observation of electrostatic fields by electron holography : The case of reverse-biased p-n junctions, Ultramicroscopy 23 (1) (1987) 29-37. doi :10.1016/0304-3991(87)90224-5.

URL http://www.sciencedirect.com/science/article/pii/0304399187902245

[25] G. Matteucci, G. F. Missiroli, J. W. Chen, G. Pozzi, Mapping of microelectric and magnetic fields with double-exposure electron holography, Applied Physics Letters 52 (3) (1988) 176-178. doi :10.1063/1.99511.

URL http://scitation.aip.org/content/aip/journal/apl/52/3/10.1063/1.99511

[26] G. Matteucci, G. Missiroli, E. Nichelatti, A. Migliori, M. Vanzi, G. Pozzi, Electron holography of long-range electric and magnetic fields, Journal of applied physics 69 (4) (1991) 1835-1842.

URL http://scitation.aip.org/content/aip/journal/jap/69/4/10.1063/1.348970

[27] G. Pozzi, Electron holography of long-range electromagnetic fields : A tutorial, in : G. C. Peter W. Hawkes, Pier Georgio Merli, M. VittoriAntisari (Eds.), Advances in Imaging and Electron Physics, Vol. Volume 123 of Microscopy, Spectroscopy, Holography and Crystallography with Electrons, Elsevier, 2002, pp. 207-223.

URL http://www.sciencedirect.com/science/article/pii/S1076567002800647

[28] Q. Ru, J. Endo, A. Tonomura, Highly sensitive moire technique for direct and real-time observation of electron microscopic phase objects, Applied 
Physics Letters 60 (23) (1992) 2840-2842. doi :10.1063/1.106841.

URL http://scitation.aip.org/content/aip/journal/apl/60/23/10.1063/1.106841

[29] A. Ohshita, M. Okuhara, C. Matsuya, K. Hata, K. Iida, Direct visualization of electromagnetic microfields by superposition of two kinds of electron holograms, Microchimica Acta 155 (1-2) (2006) 225-228. doi :10.1007/s00604-006-0547-4.

URL http://link. springer.com/article/10.1007/s00604-006-0547-4

[30] T. Hirayama, G. Lai, T. Tanji, N. Tanaka, A. Tonomura, Interference of three electron waves by two biprisms and its application to direct visualization of electromagnetic fields in small regions, Journal of Applied Physics 82 (2) (1997) 522-527. doi :10.1063/1.365610.

URL http://scitation.aip.org/content/aip/journal/jap/82/2/10.1063/1.365610

[31] K. Miyashita, K. Yamamoto, T. Hirayama, T. Tanji, Direct observation of electrostatic microfields by four-electron-wave interference using two electron biprisms, Journal of Electron Microscopy 53 (6) (2004) 577-582. doi :10.1093/jmicro/dfh074.

URL http://jmicro.oxfordjournals.org/content/53/6/577

[32] SMIGIELSKI Paul, Interférométrie holographique principes, Techniques de l'ingénieur Optique physique base documentaire : TIB528DUO (ref. article : af3345).

URL http://www . techniques-ingenieur.fr/base-documentaire/sciences-fondamentales-th8/opt

[33] F. Hue, C. L. Johnson, S. Lartigue-Korinek, G. Wang, P. R. Buseck, 
M. J. Hytch, Calibration of projector lens distortions, Journal of Electron Microscopy 54 (3) (2005) 181-190. doi :10.1093/jmicro/dfi042.

URL http://jmicro.oxfordjournals.org/cgi/doi/10.1093/jmicro/dfi042

[34] J. F. Einsle, C. Gatel, A. Masseboeuf, R. Cours, M. A. Bashir, M. Gubbins, R. M. Bowman, E. Snoeck, In situ electron holography of the dynamic magnetic field emanating from a hard-disk drive writer, Nano Research (2014) 1-9doi :10.1007/s12274-014-0610-0.

URL http://link.springer.com/article/10.1007/s12274-014-0610-0

[35] L. de Knoop, F. Houdellier, C. Gatel, A. Masseboeuf, M. Monthioux, M. Hÿtch, Determining the work function of a cold field emitter by modeling and in situ electron holography, Micron In press.

[36] E. Volkl, L. F. Allard, D. C. Joy, Introduction to Electron Holography, Kluwer Academic/Plenum Publishers, New York, 1999.

[37] J. S. Kim, T. LaGrange, B. W. Reed, M. L. Taheri, M. R. Armstrong, W. E. King, N. D. Browning, G. H. Campbell, Imaging of transient structures using nanosecond in situ tem, Science 321 (5895) (2008) 1472-1475.

[38] A. H. Zewail, Four-Dimensional Electron Microscopy, Science 328 (5975) (2010) 187-193. doi :10.1126/science.1166135.

URL http://www.sciencemag.org/cgi/content/abstract/328/5975/187 